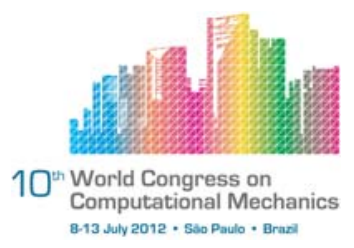

\title{
A VARIATIONAL APPROACH FOR FIBER REINFORCED VISCOELASTIC MATERIALS SUBJECT TO DAMAGE
}

\author{
E. A. Fancello ${ }^{1}$, J. M. Vassoler ${ }^{2}$, A. E. Selke ${ }^{3}$, L. Stainier ${ }^{3}$ \\ ${ }^{1}$ Department of Mechanical Engineering, Federal University of Santa Catarina, Brazil \\ (fancello@grante.ufsc.br) \\ ${ }^{2}$ Department of Mechanical Engineering, Federal University of Rio Grande do Sul, Brazil \\ ${ }^{3}$ Institut GeM, Ecole Centrale de Nantes, Nantes, France
}

\begin{abstract}
A variational approach for fiber reinforced viscoelastic materials subject to damage The mechanical properties of soft biological tissues vary depending on how the internal structure is organized. Classical examples of tissues are ligaments, tendons, skin, arteries, and annulus fibrous. The main element of such tissues is the fibers which are responsible for the tissue resistance and the main mechanical characteristic is their viscoelastic anisotropic behavior. The objective of this paper is to extend an existing model for viscoelastic materials with (anisotropic) reinforcement of fibers in order to include damage that arises when strains assumes values greater than some physiological range. The model is based on a variational framework in which its mechanical behavior is described by a free energy incremental potential whose local minimization provides the constraints for the internal variable updates for each load increment. Among the advantages of these variational approaches we should mention the ability to represent different material models depending on the choice of suitable potential functions and the obtention of symmetric constitutive tangent matrices. Some numerical examples are shown in order to evaluate the performance of the proposed model.
\end{abstract}

Keywords: Viscoelasticity, Damage, Finite strains.

\section{INTRODUCTION}

In this work is proposed a variational constitutive model appropriate to simulate the mechanical behaviour of connective soft tissues (e.g. ligaments and tendons) subject to large strains, different loading velocities and damage. These soft biological tissues are mainly formed by arrangements of collagen fibers embedded in a cellular matrix. This internal structure provides an anisotropic mechanical response dependent on the fiber directions as well as a viscosity due to intersticial fluid and interaction among fibers. Many models have been proposed to model these kinds of materials relating different phenomena (large strains, hyperelasticity, anisotropy, viscosity, damage, etc.). Among them, we can mention [1],[2],[3],[4],[5],[6]. 
In [7] a variational framework for viscoelastic anisotropic materials submitted to finite strain regime is proposed. The aim of the present work is to extend this variational formulation to include damage behaviours, including different damage equations proposed in literature ([3], [5], [4],[6]).

The variational framework of this paper is stated in Section 2. Section 3 presents the variational damage formulation. Section 4 shows some preliminary results using different damage functions proposed in literature. Final remarks are enclosed in Section 6.

\section{VARIATIONAL CONSTITUTIVE MODEL}

Hyperelastic models are based on the existence of a free energy function $W$ which is dependent only on the total strain. Then, the first Piola-Kirchhoff stress tensor $\mathbf{P}$ is defined as

$$
\mathbf{P}=\frac{\partial W(\mathbf{F})}{\partial \mathbf{F}}=2 \mathbf{F} \frac{\partial W(\mathbf{C})}{\partial \mathbf{C}}
$$

where $\mathbf{F}$ is the gradient of deformation and $\mathbf{C}=\mathbf{F}^{T} \mathbf{F}$ the Cauchy-Green tensor.

The stress state of dissipative materials is dependent not only on the total strain but also on the strain history. In order to overcome this difficulty an approach based on variational concepts was proposed in [8] in which the constitutive problem can be stated analogously to Eq.(1) in an incremental way. Variational constitutive models are based on pseudohyperelastic potentials where the constitutive problem can be stated analogously to a hyperelastic model. In this approach a pseudo-potential energy, also called the Incremental Potential, is defined at each load step, providing the first Piola-Kichhoff stress as follows:

$$
\mathbf{P}_{n+1}=\frac{\partial \Psi\left(\mathbf{F}_{n+1} ; \xi_{n}\right)}{\partial \mathbf{F}_{n+1}}=2 \mathbf{F}_{n+1} \frac{\partial \Psi\left(\mathbf{C}_{n+1} ; \xi_{n}\right)}{\partial \mathbf{C}_{n+1}}
$$

In this expression, $\xi=\left\{\mathbf{F}, \mathbf{F}^{i}, \mathbf{Q}\right\}$ is the set of external and internal state variables. The elastic and inelastic gradients of deformation $\mathbf{F}^{e}$ and $\mathbf{F}^{i}$ are obtained from the multiplicative decomposition of $\mathbf{F}$. The symbol $\mathbf{Q}$ includes all remaining internal variables related to the dissipative phenomena. In [8] it is shown that the Incremental Potential may have the expression:

$$
\begin{gathered}
\Psi\left(\mathbf{F}_{n+1} ; \xi_{n}\right)=\min _{\mathbf{F}_{n+1}^{i}, \mathbf{Q}_{n+1}}\left\{W\left(\xi_{n+1}\right)-W\left(\xi_{n}\right)+\Delta t \psi\left(\stackrel{\circ}{\mathbf{F}}^{i}, \stackrel{\mathbf{Q}}{\xi_{n}}\right)\right\} \\
W(\xi)=\varphi(\mathbf{F})+\varphi^{e}\left(\mathbf{F F}^{i^{-1}}\right)+\varphi^{i}\left(\mathbf{F}^{i}, \mathbf{Q}\right)
\end{gathered}
$$

The strain energy is decomposed additively into elastic and inelastic contributions $\varphi, \varphi^{e}$ and $\varphi^{i}$, depending on the total value of $\mathbf{F}$, on the elastic part $\mathbf{F}^{e}$ and on the inelastic part $\mathbf{F}^{i}$ and internal variables $\mathbf{Q}$ respectively. $\psi$ is the (pseudo) potential that provides the dependence of the stress on the rate (incremental approximation of rate) variables $\mathbf{F}^{i}$ and $\mathbf{Q}$.

The minimization problem (3) identifies the optimal values of $\mathbf{F}_{n+1}^{i}$ and $\mathbf{Q}_{n+1}$, which define the internal variables associated with the new state $\mathbf{F}_{n+1}$. Once this minimization problem is solved, stresses may be computed by Eq.(2) as in hyperelastic models. Different material models may be constructed in this general framework depending on the particular choices and arrangements of potentials $\varphi, \varphi^{e}, \varphi^{i}$ and $\psi$. 


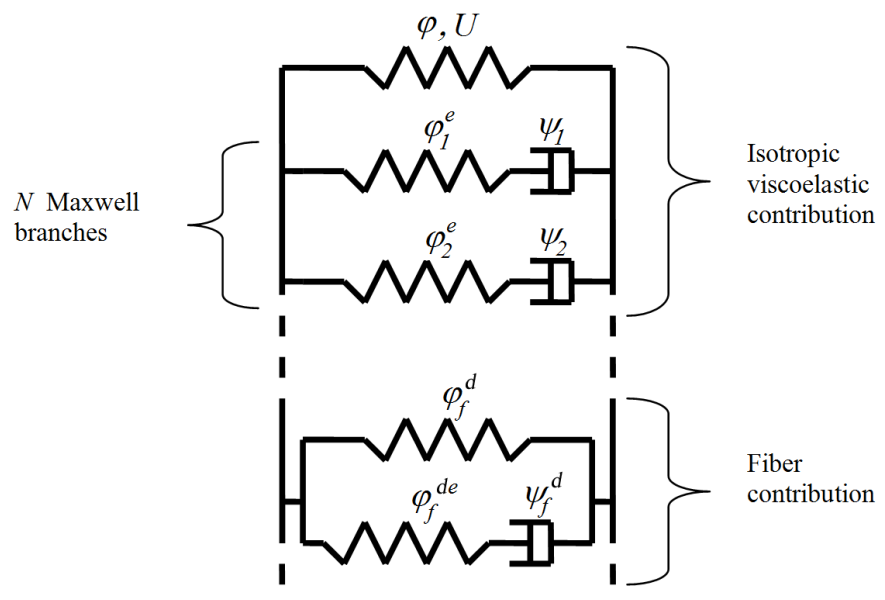

Figure 1. Rheological model

\section{ANISOTROPIC VISCOELASTIC MODEL SUBJECT TO DAMAGE}

The anisotropic viscoelastic damage model proposed here is an extension of the work [7] in order to introduce mechanical damage on the fiber-reinforcement, which became significant on finite strains. The inclusion of fibers is performed following concepts shown in [9] by an additive decomposition of energies:

$$
\Psi=\Psi_{i s o}+\Psi_{f}^{d}
$$

where, $\Psi_{i s o}$ is the potential related to the isotropic viscoelastic model proposed in [10], while $\Psi_{f}^{d}$ is related to the fiber contribution subject to mechanical damage.

The fiber-reinforcement contribution proposed in [7], that represents the anisotropic fiber behaviour, is modified to take into account the mechanical damage.

Figure 1 shows a rheological representation of the addition (5) in which both the isotropic and the fiber contributions are connected in parallel, reacting independently of each other for the same total strain. The additive decomposition in Equation (5) states that the incremental potential of the isotropic matrix and that of the fibers are uncoupled. The same happens for the elastic and Maxwell branches of the fiber contribution. Each branch depends on the given strain increment over $\Delta t$ and the constitutive response of the composite comes only from the additive constitutive response of each component, which is clearly illustrated in Figure 1. Moreover, this model considers that fibers are continuously distributed in the isotropic ground substance (matrix) [9] and therefore, no distinction is made on the size or length of them.

\subsection{Isotropic Incremental Potential}

As mentioned above, the incremental potential $\Psi_{i s o}$ is exactly that presented in [10]. Nevertheless, we use this section to make a brief description of this model in order to keep the text minimally self-contained. We begin by assuming a classical multiplicative decomposition of the gradient of deformation into isochoric and volumetric parts $\mathbf{F}=J \hat{\mathbf{F}}$ with $J=\operatorname{det}(\mathbf{F})$. The isochoric part allows also for a multiplicative separation into elastic and 
viscous contributions: $\hat{\mathbf{F}}^{i}=\hat{\mathbf{F}}^{e} \mathbf{F}^{v}$. With these hypotheses, the free energy ${ }^{1} W$ is defined as

$$
W(\mathbf{C})=U(J)+\varphi(\hat{\mathbf{C}})+\varphi^{e}\left(\hat{\mathbf{C}}^{e}\right)
$$

where

$$
\hat{\mathbf{C}}=\hat{\mathbf{F}}^{T} \hat{\mathbf{F}} \quad \hat{\mathbf{C}}^{e}=\hat{\mathbf{F}}^{e^{T}} \hat{\mathbf{F}}^{e}
$$

The isochoric potential $\varphi$ is an isotropic function of the eigenvalues $c_{i}$ of $\mathbf{C}$. The (elastic) volumetric contribution originates from the potential $U$ which is dependent on $J$ :

$$
U(J)=\frac{k}{2}[\ln J]^{2}
$$

The potentials $\varphi, \varphi^{e}$ and $\psi$ are assumed to be isochoric functions of the Cauchy tensors $\hat{\mathbf{C}}, \hat{\mathbf{C}}^{e}$ and of the viscous stretching $\mathbf{D}^{v}$ by means of their respective eigenvalues $c_{i}, c_{i}^{e}$ and $d_{i}^{v}$, i.e., $\varphi(\hat{\mathbf{C}})=\varphi\left(c_{1}, c_{2}, c_{3}\right), \varphi^{e}\left(\hat{\mathbf{C}}^{e}\right)=\varphi\left(c_{1}^{e}, c_{2}^{e}, c_{3}^{e}\right), \psi\left(\mathbf{D}^{v}\right)=\psi\left(d_{1}^{v}, d_{2}^{v}, d_{3}^{v}\right)$.

The viscous stretching $\mathbf{D}^{v}$ is defined by

$$
\mathbf{D}^{v}=\operatorname{Sym}\left(\mathbf{L}^{v}\right)=\mathbf{L}^{v}=\dot{\mathbf{F}}^{v} \mathbf{F}^{v^{-1}}
$$

where it is defined that $\mathbf{L}^{v}$ is symmetric. The viscous flow is assumed to be isochoric by means of the following constrains on the spectral components of $\mathbf{D}^{v}$

$$
\begin{aligned}
d_{j}^{v} \in K_{Q} & =\left\{p_{j} \in \mathbb{R} \Rightarrow p_{1}+p_{2}+p_{3}=0\right\} \\
\mathbf{M}_{j}^{v} \in K_{M} & =\left\{\mathbf{N}_{j} \in \operatorname{Sym} \Rightarrow \mathbf{N}_{j} \cdot \mathbf{N}_{j}=1, \mathbf{N}_{i} \cdot \mathbf{N}_{j}=0, i \neq j\right\}
\end{aligned}
$$

From these definitions, it is shown in [10] that at each time increment $\Delta t$, the isotropic Incremental Potential in Eq.(3), takes the form

$$
\Psi_{i s o}\left(\mathbf{F}_{n+1} ; \xi_{n}\right)=\Delta \varphi\left(\hat{\mathbf{C}}_{n+1}\right)+\Delta U\left(J_{n+1}\right)+\min _{\mathbf{M}_{j}^{v}, \Delta q_{j}^{v}}\left\{\Delta \varphi^{e}\left(\hat{\mathbf{C}}_{n+1}^{e}\right)+\Delta t \psi\left(\mathbf{D}_{n+1}^{v}\right)\right\}
$$

subject to

$$
\begin{aligned}
& d_{j}^{v}=\frac{\Delta q_{j}^{v}}{\Delta t}, \quad \Delta q_{j}^{v} \in K_{Q}=\left\{p_{j} \in \mathbb{R} \Rightarrow p_{1}+p_{2}+p_{3}=0\right\} \\
& \mathbf{M}_{j}^{v} \in K_{M}=\left\{\mathbf{N}_{j} \in \mathrm{Sym} \Rightarrow \mathbf{N}_{j} \cdot \mathbf{N}_{j}=1, \mathbf{N}_{i} \cdot \mathbf{N}_{j}=0, i \neq j\right.
\end{aligned}
$$

In expression (11) we have

$$
\begin{aligned}
\Delta \varphi\left(\hat{\mathbf{C}}_{n+1}\right) & =\varphi\left(\hat{\mathbf{C}}_{n+1}\right)-\varphi\left(\hat{\mathbf{C}}_{n}\right) \\
\Delta \varphi^{e}\left(\hat{\mathbf{C}}_{n+1}^{e}\right) & =\varphi^{e}\left(\hat{\mathbf{C}}_{n+1}^{e}\right)-\varphi^{e}\left(\hat{\mathbf{C}}_{n}^{e}\right) \\
\Delta U\left(J_{n+1}\right) & =U\left(J_{n+1}\right)-U\left(J_{n}\right)
\end{aligned}
$$

with

$$
\begin{gathered}
\hat{\mathbf{C}}_{n+1}^{e}=\hat{\mathbf{F}}_{n+1}^{e^{T}} \hat{\mathbf{F}}_{n+1}^{e}=\hat{\mathbf{C}}^{p r}\left(\exp \left[\Delta t \mathbf{D}_{n+1}^{v}\right]\right)^{-2} \\
\hat{\mathbf{C}}^{p r}=\mathbf{F}_{n}^{v^{-T}} \hat{\mathbf{C}}_{n+1} \mathbf{F}_{n}^{v^{-1}} \quad \Delta t \mathbf{D}_{n+1}^{v}=\sum_{j=1}^{3} \Delta q_{j}^{v} \mathbf{M}_{j} \\
\mathbf{F}_{n+1}^{v} \mathbf{F}_{n}^{v^{-1}}=\exp \left[\Delta t \mathbf{D}_{n+1}^{v}\right] \quad \hat{\mathbf{F}}_{n+1}^{e}=\hat{\mathbf{F}}_{n+1} \hat{\mathbf{F}}_{n+1}^{v^{-1}}
\end{gathered}
$$

\footnotetext{
${ }^{1}$ For simplicity of notation we omit the subscript $i$ so from the potentials associated with the isotropic contributions. However, we maintain the subscript $f$ for the potentials associated with the fiber counterpart.
} 
The optimality condition of (11) with respect to $\mathbf{M}_{j}^{v}$ results in $\hat{\mathbf{C}}_{n+1}^{e}, \hat{\mathbf{C}}^{p r}$ and $\mathbf{D}^{v}$ sharing the same eigenvectors $\mathbf{E}_{j}^{e}=\mathbf{E}_{j}^{p r}=\mathbf{M}_{j}^{v}$ respectively. Finally, the minimization with respect to $\Delta q_{j}^{v}$ results in the following set of nonlinear equations [10]:

$$
\begin{gathered}
\frac{\partial \varphi^{e}}{\partial \varepsilon_{j}^{e}}-\frac{\partial \psi}{\partial d_{j}^{v}}+\lambda=0 \quad j=1,2,3 \\
\Delta q_{1}^{v}+\Delta q_{2}^{v}+\Delta q_{3}^{v}=0
\end{gathered}
$$

where $\lambda$ is the Lagrangian multiplier associated with the constraint (12) and $\varepsilon_{j}^{e}=\frac{1}{2} \ln \left(c_{j}^{e}\right)$. The four nonlinear equations (16) can be solved by the Newton method, a technique that also provides the analytical tangent modulus to be used in the global equilibrium problem. Once the minimizers $\Delta q_{j}^{v}$ are obtained, the first Piola-Kirchhoff stress tensor is calculated by the classic "hyperelastic-like" expression. Detailed information regarding these operations is found in [10].

\subsection{Anisotropic Viscoelastic Damage Incremental Potential}

The corresponding Incremental Potential for anisotropic damage behaviour $\Psi_{f}^{d}$, related to fiber directions, is not only dependent on the Cauchy tensor $\mathbf{C}$, but also on the structural tensor $\mathbf{A}_{f}=\mathbf{a}_{f} \otimes \mathbf{a}_{f}$, where $\mathbf{a}_{f}$ is the unit vector defining the fiber direction. This dependence in the present case is related to the invariant $I_{f}[1]$ :

$$
I_{f}=\hat{\mathbf{C}}: \mathbf{A}_{f}=\mathbf{a}_{f} \cdot \hat{\mathbf{C}} \cdot \mathbf{a}_{f}=\lambda_{f}^{2}
$$

which has the particular physical interpretation of the quadratic stretch in the direction of the fiber $\lambda_{f}^{2}$. The total elongation $\lambda_{f}$ acting on the Maxwell branch of the fiber contribution (Fig.1) may be decomposed into elastic and inelastic and viscous contributions: $\lambda_{f}=\lambda_{f}^{e} \lambda_{f}^{v}$. The rate of viscous stretching of the fiber is defined as:

$$
d_{f}^{v}=\dot{\lambda}_{f}^{v} \lambda_{f}^{v-1}
$$

The incremental evolution of the viscous stretch is obtained using the exponential mapping proposed in [11] that allows us to write

$$
\lambda_{f_{n+1}}^{v}=\exp \left(\Delta t d_{f}^{v}\right) \lambda_{f_{n}}^{v} ; \quad \Delta \lambda_{f}^{v}=\exp \left(\Delta t d_{f}^{v}\right)=\frac{\lambda_{f_{n+1}}^{v}}{\lambda_{f_{n}}^{v}} ; \quad d_{f}^{v}=\frac{1}{\Delta t} \ln \left(\Delta \lambda_{f}^{v}\right)
$$

The anisotropic viscoelastic pseudo-potential proposed by [7] carry out the dependence of an internal state variable which introduce viscoelastic effects. The fiber counterpart of the free energy $W_{f}$ is defined as

$$
W_{f}=\varphi_{f}+\varphi_{f}^{e}
$$

The potentials $\varphi_{f}$ and $\varphi_{f}^{e}$ are assumed to be functions of the total fiber stretch $\lambda_{f}$ and of the elastic fiber stretch of the Maxwell branch $\lambda_{f}^{e}$, respectively. It is also assumed the existence of a dissipative pseudo-potential $\psi_{f}$ that is function of the rate of fiber viscous stretching $d_{f}^{v}$ :

$$
\varphi_{f}=\varphi_{f}\left(\lambda_{f}\right) ; \quad \varphi_{f}^{e}=\varphi_{f}^{e}\left(\lambda_{f}^{e}\right) ; \quad \psi_{f}=\psi_{f}\left(d_{f}^{v}\right)
$$

In order to include the phenomenon of fiber damaging in the model proposed in [7], two new internal variables $\eta$ and $\eta^{e}$ are added. This changes the expressions of the elastic 
potentials during the deformation process and consequently the mechanical behaviour of the material. $\eta$ is the internal damage variable that acts on the potential $\varphi_{f}$ of the main branch of the fiber contribution while $\eta^{e}$ is the internal damage variable that acts on the potentials $\varphi_{f}^{e}$ and $\psi_{f}$ of the Maxwell branch of the fiber contribution. A simple form for this inclusion is the following:

$$
W_{f}=\varphi_{f}^{d}+\varphi_{f}^{e d}
$$

where

$$
\begin{gathered}
\varphi_{f}^{d}\left(\lambda_{f}, \eta\right)=(1-\eta) \varphi_{f}\left(\lambda_{f}\right)+\phi(\eta) \quad \chi_{f}=\chi_{f}(\dot{\eta}) \\
\varphi_{f}^{e d}\left(\lambda_{f}^{e}, \eta^{e}\right)=\left(1-\eta^{e}\right) \varphi_{f}^{e}\left(\lambda_{f}^{e}\right)+\phi^{e}\left(\eta^{e}\right) \quad \chi_{f}^{e}=\chi_{f}^{e}\left(\dot{\eta}^{e}\right)
\end{gathered}
$$

The potentials $\phi$ and $\phi^{e}$ account for the laws of hardenning/softenning of the fibers due to the mechanical damage. $\chi_{f}$ and $\chi_{f}^{e}$ are dissipative pseudo-potentials depending on the rate of the corresponding damage variables and intimately related tho their evolution.

With these definitions and following analogous arguments found in [10] and [7] it is defined the following fiber contribution of the incremental potential:

$$
\begin{aligned}
\Psi_{f}^{d} & =\min _{\eta_{n+1}}\left\{\Delta \varphi_{f}^{d}\left(\lambda_{f_{n+1}}, \eta_{n+1}\right)+\Delta t \chi_{f}\left(\dot{\eta}_{n+1}\right)\right\}+ \\
& +\min _{\lambda_{f_{n+1}}^{v}, \eta_{n+1}^{e}}\left\{\Delta \varphi_{f}^{e d}\left(\lambda_{f_{n+1}}^{e}\left(\lambda_{f_{n+1}}^{v}\right), \eta_{n+1}^{e}\right)+\Delta t \psi_{f}\left(d_{f_{n+1}}^{v}\left(\lambda_{f_{n+1}}^{v}\right)\right)+\Delta t \chi_{f}^{e}\left(\dot{\eta}_{n+1}^{e}\right)\right\}
\end{aligned}
$$

where

$$
\begin{array}{r}
\Delta \varphi_{f}^{d}=\varphi_{f}^{d}\left(\lambda_{f_{n+1}}, \eta_{n+1}\right)-\varphi_{f}^{d}\left(\lambda_{f_{n}}, \eta_{n}\right) \\
\Delta \varphi_{f}^{e d}=\varphi_{f}^{e d}\left(\lambda_{f_{n+1}}^{e}, \eta_{n+1}^{e}\right)-\varphi_{f}^{e d}\left(\lambda_{f_{n}}^{e}, \eta_{n}^{e}\right)
\end{array}
$$

Physically, the fibers do not bear compressive stresses. Therefore, potentials $\varphi_{f}, \varphi_{f}^{e}$ and $\psi_{f}$ have some particularities, which leads to some care being required in approaching the minimization problem. This is discussed in detail in [7].

\subsection{Material Models}

The mechanical behaviour of different materials may be represented from a suitable choice of the potential functions. Those used in the present article are shown bellow.

\subsubsection{Hencky and Ogden Models}

The Hencky expressions are of the type

$$
\varphi=\mu \sum_{j=1}^{3}\left(\ln \left(\lambda_{j}\right)\right)^{2}, \quad \varphi^{e}=\mu^{e} \sum_{j=1}^{3}\left(\ln \left(\lambda_{j}^{e}\right)\right)^{2}, \quad \psi=\eta^{v} \sum_{j=1}^{3}\left(d_{j}^{v}\right)^{2}
$$

while the Ogden expressions are written as

$$
\begin{aligned}
\varphi & =\sum_{j=1}^{3} \sum_{p=1}^{N} \frac{\mu_{p}}{\alpha_{p}}\left(\left[\lambda_{j}\right]^{\alpha_{p}}-1\right), \quad \varphi^{e}=\sum_{j=1}^{3} \sum_{p=1}^{N} \frac{\mu_{p}^{e}}{\alpha_{p}}\left(\left[\lambda_{j}^{e}\right]^{\alpha_{p}}-1\right), \\
\psi & =\sum_{j=1}^{3} \sum_{p=1}^{N} \frac{\eta_{p}^{v}}{\alpha_{p}}\left(\left[d_{j}^{v}\right]^{\alpha_{p}}-1\right) .
\end{aligned}
$$

Symbols $\mu, \mu^{e}, \eta^{v}, \mu_{p}, \mu_{p}^{e}, \mu_{p}^{v}, \alpha_{p}$ are material parameters to be identified. More details on this issue are found in [10]. 


\subsubsection{Fiber Material Models}

Different expressions of hyperelastic fiber-materials are found in the literature. In this work is used that proposed in [1]:

$$
\varphi_{f}=\left\{\begin{array}{rll}
\frac{k_{1}}{2 k_{2}}\left\{\exp \left[k_{2}\left(\lambda_{f}^{2}-1\right)^{2}\right]-1\right\} & \text { if } & \lambda_{f}>0 \\
0 & \text { if } & \lambda_{f} \leq 0
\end{array}\right.
$$

Similar behaviour is set for the dissipative potential $\psi_{f}$, attributing a zero value to a compressive viscous stretching:

$$
\psi_{f}\left(d_{f}^{v}\right)=\left\{\begin{array}{rll}
\bar{\psi}_{f}\left(d_{f}^{v}\right) & \text { if } & d_{f}^{v} \geq 0 \\
0 & \text { if } & d_{f}^{v}<0
\end{array}\right.
$$

where $\bar{\psi}_{f}$ is any of the viscous models previously presented (Hencky or Ogden Model).

\subsubsection{Damage Material Models}

Different functions has been proposed in the literature for the damage variable. Consider first the following intermediate variables

$$
\Phi^{d}=\sqrt{\max _{s \in(-\infty, t)} \varphi_{f}\left(\lambda_{f}(s)\right)}, \quad \Lambda=\frac{\Phi^{d}-\Phi_{\min }^{d}}{\Phi_{\max }^{d}-\Phi_{\min }^{d}}
$$

where $\Phi^{d}$ has the meaning of the square root of the maximum value the strain energy of the undamaged fiber achieved in time, $\alpha, \beta, \Phi_{\min }^{d}$ and $\Phi_{\max }^{d}$ are material parameters and $\Lambda$ is a normalization of $\Phi^{d}$. In [3] is proposed the following exponential expression of damage as a function of $\Phi^{d}$ :

$$
\eta\left(\Phi^{d}\right)=\left\{\begin{array}{l}
0 \quad \text { if } \quad \Phi^{d}<\Phi_{\min }^{d} \\
1-\frac{1-\exp \left(\beta\left[\Phi^{d}-\Phi_{\max }^{d}\right]\right)}{1-\exp \left(\beta\left[\Phi_{\min }^{d}-\Phi_{\max }^{d}\right]\right)} \text { if } \quad \Phi_{\min }^{d} \leq \Phi^{d} \leq \Phi_{\max }^{d} \\
1 \quad \text { if } \quad \Phi^{d}>\Phi_{\max }^{d}
\end{array}\right.
$$

In [4], similar expression is proposed, now of a polynomial type:

$$
\eta\left(\Phi^{d}\right)= \begin{cases}0 & \text { if } \quad \Phi^{d}<\Phi_{\min }^{d} \\ \Lambda^{2}\left[1-\beta\left[\Lambda^{2}-1\right]\right] \text { if } \quad \Phi_{\min }^{d} \leq \Phi^{d} \leq \Phi_{\max }^{d} \\ 1 \quad \text { if } \quad \Phi^{d}>\Phi_{\max }^{d}\end{cases}
$$

In [5] takes the form

$$
\eta\left(\Phi^{d}\right)=\frac{1}{2}\left[1+\frac{\alpha \Phi^{d} \exp \left[2 \alpha\left(\frac{2 \Phi^{d}}{\beta}-1\right)\right]-1}{\alpha \Phi^{d} \exp \left[2 \alpha\left(\frac{2 \Phi^{d}}{\beta}-1\right)\right]+1}\right]
$$

while in [6] a mathematical function of " $\mathrm{S}$ " shape is stated:

$$
\eta\left(\Phi^{d}\right)=\frac{1}{1+\exp \left(-\alpha\left[\Phi^{d}-\beta\right]\right)}
$$


All these cases correspond to pure elastic-damage models in which the damage variable $\eta$ is completely defined by the current value of $\Phi^{d}$.

In the present framework, $\eta$ is obtained from the optimality condition (25) in wich a potential function $\phi(\eta)$ is needed (see (23) and (25)). To obtain this potential function it is possible to use an approach similar to that presented by [12]. The expressions given above were all proposed in the context of elastic-damage models with a strain-energy density function of the form

$$
\Psi(\mathbf{F}, \eta)=\eta \Psi(\mathbf{F})+\phi(\eta)
$$

where it is possible to obtain the function $\frac{\partial \phi(\eta)}{\partial \eta}$ using the constrain (See [12]):

$$
\frac{\partial \Psi}{\partial \eta}=0
$$

which results in the following expression:

$$
\frac{\partial \phi(\eta)}{\partial \eta}=\Psi(\mathbf{F})
$$

The expression (39) may be used to obtain the correspond function $\phi(\eta)$ of each damage expression $\eta\left(\Phi^{d}\right)$ presented above for elastic-damage models. It should be noted that the constraint (38) is conceptually analogous to the optimality conditions of the minimization problem (25) with respect to $\eta$. The difference appears due to the dissipative potential $\chi_{f}$ that characterizes the possible dependence of the process to the rate $\dot{\eta}$ :

$$
\chi_{f}(\dot{\eta})=\left\{\begin{array}{rll}
\bar{\chi}_{f}(\dot{\eta}) & \text { if } & \dot{\eta} \geq 0 \\
0 & \text { if } & \dot{\eta}<0
\end{array}\right.
$$

\section{PRELIMINARY RESULTS}

In order to verify the ability of the proposed model to represent the viscoelasticdamage behaviour, uniaxial stretching tests with controlled stretching cycles are performed. Since the anisotropic behaviour was extensively investigated in [7], here only the damage effect on the fiber direction is presented.

The material parameters are presented in Table 1. These parameters don't have any correlation with a real materials and have been chosen to study the model capability. In this section are presented only results for the damage models proposed by [5] and [4], respectivelly, Case I and Case II.

The results using the damage function correspondent to the Case I are presented in Fig.2-4. Fig. 2 presents the stress response of the model. Fig. 3 shown separately the stress contribution of the fiber Main branch and the fiber Maxwell branch. Finally, Fig.4 presents the history of the damage variables $\eta$ (Main branch) and $\eta^{e}$ (Maxwell branch).

The results using the damage function correspondent to the Case II are presented in Fig.5-7. In the same order proposed above, Fig.5 presents the stress response of the model, Fig. 6 shown separately the stress contribution of the fiber main branch and the fiber Maxwell's branch and Fig.7 presents the history of the damage variables $\eta$ (Main branch) and $\eta^{e}$ (Maxwell's branch). 
Table 1. Material parameters used on the uniaxial tests for the fiber contribution.

\begin{tabular}{|c|c|c|}
\hline Potential & \multicolumn{2}{|c|}{ Fiber } \\
\hline & Model & Parameters \\
\cline { 2 - 3 }$\varphi_{f}$ & Holzapfel & $k_{1}=100 \mathrm{MPa}$ e $k_{2}=1.5 \mathrm{MPa}$ \\
$\varphi_{f}^{e}$ & Holzapfel & $k_{1}=100 \mathrm{MPa}$ e $k_{2}=1.5 \mathrm{MPa}$ \\
\hline$\phi(\mathrm{I})$ & Rodriguez & $\alpha=0.1 \mathrm{e} \beta=1$ \\
$\phi^{e}(\mathrm{I})$ & Rodriguez & $\alpha=0.1 \mathrm{e} \beta=1$ \\
\hline$\phi(\mathrm{II})$ & Peña & $\beta=1, \Phi_{\min }^{d}=0$ e $\Phi_{\max }^{d}=10$ \\
$\phi^{e}(\mathrm{II})$ & Peña & $\beta=1, \Phi_{\min }^{d}=0 \mathrm{e} \Phi_{\max }^{d}=10$ \\
\hline$\psi_{f}$ & Hencky & $\eta^{v}=3000 \mathrm{MPa} \cdot \mathrm{s}^{-1}$ \\
\hline
\end{tabular}

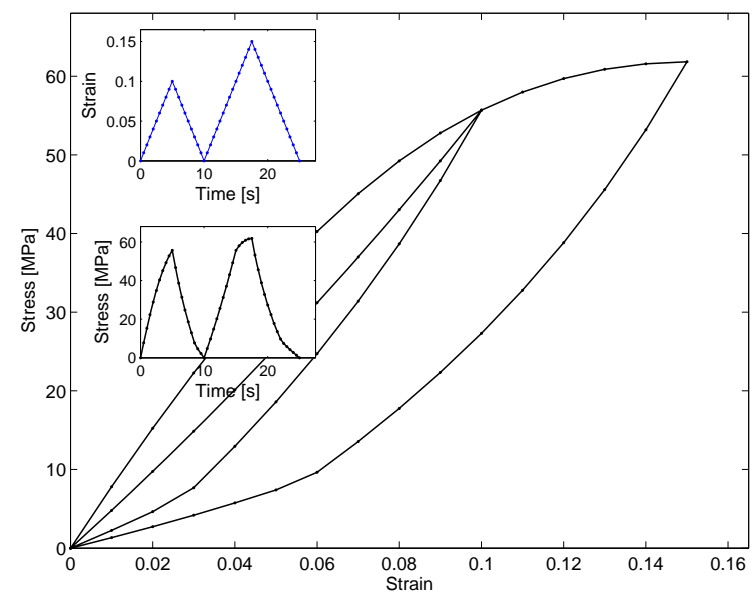

Figure 2. Stress response (Case I)

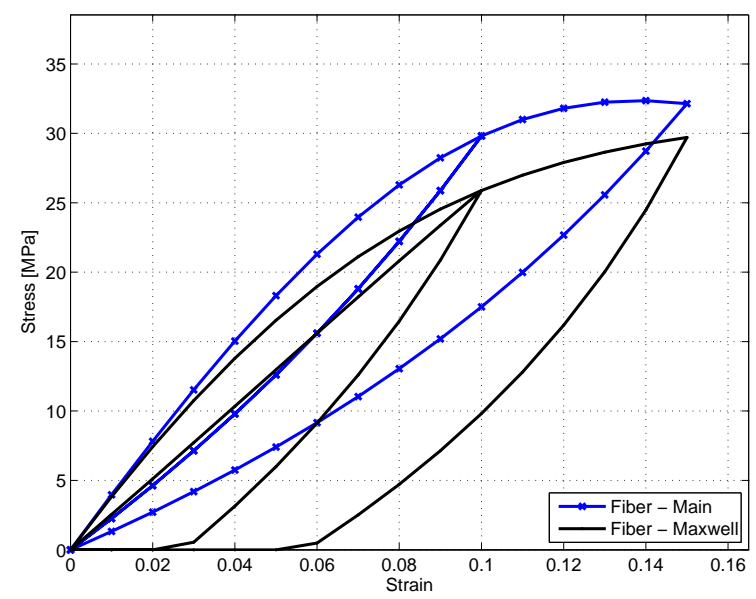

Figure 3. Stress contributions (Case I) 


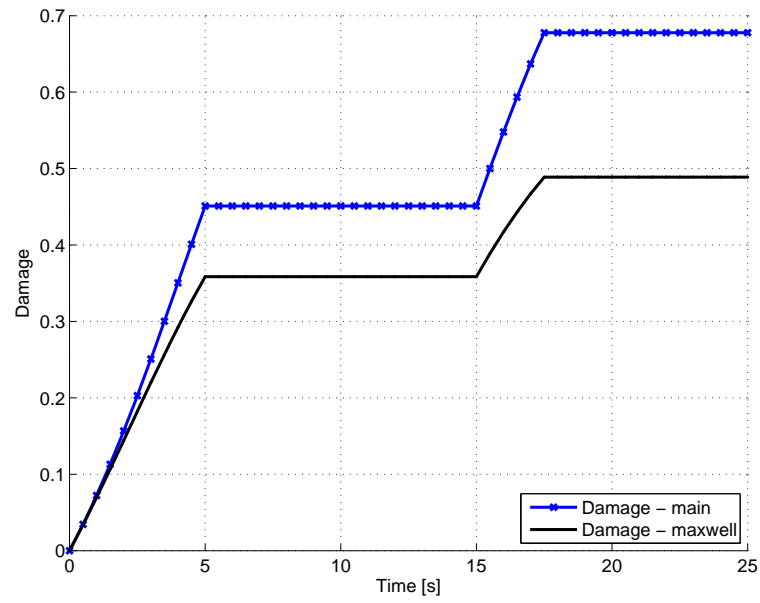

Figure 4. Damage history (Case I)

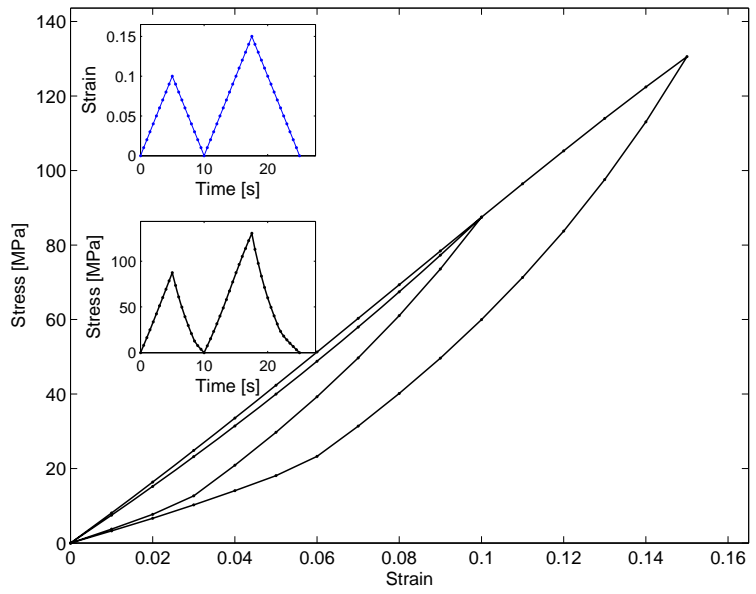

Figure 5. Stress response (Case II) 


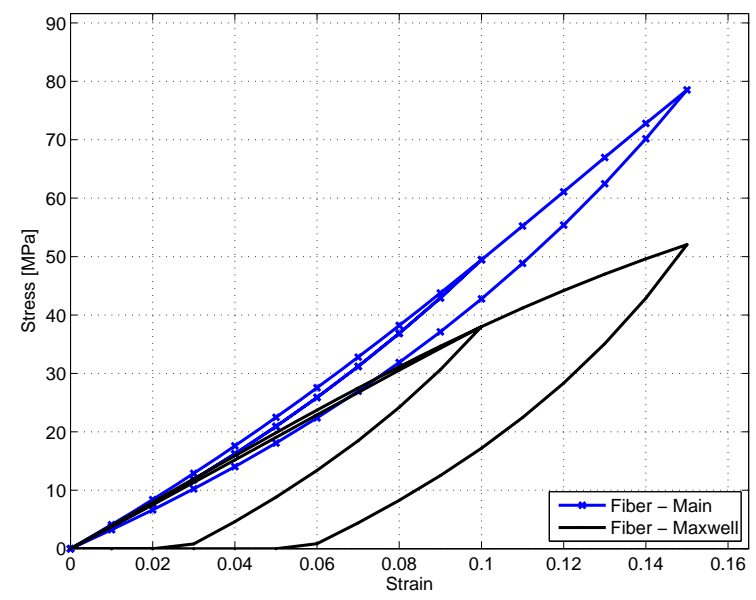

Figure 6. Stress contributions (Case II)

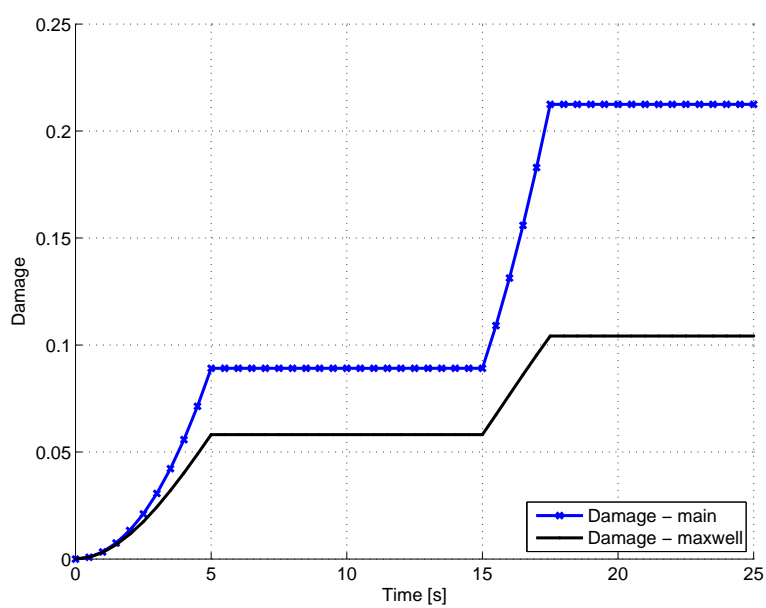

Figure 7. Damage history (Case II) 
In both cases (Case I and II) were used the same parameters in the potentials of the Main branch $\left(\varphi_{f}\right.$ and $\left.\phi\right)$ and of the Maxwell's branch $\left(\varphi_{f}^{e}\right.$ and $\left.\phi^{e}\right)$. The viscous effect is clear noted only on the Maxwell's contribution (See Fig.3 and Fig.6), while the damage behavior can be seen on both curves. The damage behavior is noticed on the begging of the second loading, where the stiffness is lower than in the first loading, and also in dissipation behavior characterized by the path of the unloading curves.

\section{CONCLUSIONS}

The main objective of this works was achieved. In the preliminary results was shown that was possible to represent viscoelastic-damage behavior by means of a convenient introduction of two new damage variables in a existent variational framework. In this case was used a fiber-reinforced viscoelastic variational model.

It should be mentioned that the presented examples have the goal of verifying the ability of the proposed approach to follow expected qualitative behaviors. Experimental data should be used in further works in order to perform a parameter identification for the proposed potentials.

\section{Acknowledgements}

Eduardo Alberto Fancello and Jakson Manfredini Vassoler thank the Brazilian "Conselho Nacional de Desenvolvimento Científico e Tecnológico" who provided financial support for this research.

\section{REFERENCES}

\section{References}

[1] Holzapfel G, Gasser TC. A viscoelastic model for fiber-reinforced composites at finite strains: Continuum basis, computational aspects and applications. Computer Methods in Applied Mechanics and Engineering 2001; 190(34):4379-4403.

[2] Limbert G, Taylor M, Middleton J. Three-dimensional finite element modelling of the human acl: simulation of passive knee flexion with a stressed and stress-free acl. Journal of Biomechanics 2004; 37(11):1723-1731.

[3] Calvo B, Peña E, Martinez MA, Doblaré M. An uncoupled directional damage model for fibred biological soft tissues. Formulation and computational aspects. International Journal for Numerical Methods in Engineering 2007; 69(10):2036-2057.

[4] Peña E, Calvo B, Martinez MA, Doblaré M. On finite-strain damage of viscoelasticfibred materials. Application to soft biological tissues. International Journal for Numerical Methods in Engineering 2008; 74(7):1198-1218.

[5] Rodriguez JF, Alustre V, Doblaré M. Finite element implementation of a stochastic three dimensional finite-strain damage model for fibrous soft tissue. Application to soft biological tissues. Computer Methods in Applied Mechanics and Engineering 2008; 197(9120:946-958. 
[6] Peña E. Damage functions of the internal variables for soft biological fibred tissues. Mechanics Research Communications 2008; 38(8):610-615.

[7] Vassoler JM, Reips L, Fancello EA. A variational framework for fiber-reinforced viscoelastic soft tissues. International Journal for Numerical Methods in Engineering 2012; 89(13):1691-1706.

[8] Ortiz M, Stainier L. The variational formulation of viscoplastic constitutive updates. Computer Methods in Applied Mechanics and Engineering 1999; 171(3-4):419-444.

[9] Holzapfel GA. Nonlinear solid mechanics: a continuum approach for engineering Wiley, 2000.

[10] Fancello EA, Ponthot JP, Stainier L. A variational formulation of constitutive models and updates in nonlinear finite viscoelasticity. International Journal for Numerical Methods in Engineering 2006; 65(11):1831-1864.

[11] Anand L, Weber G. Finite deformations constitutive equations and a time integration procedure for isotropic hyperelastic-viscoplastic solids. Computer Methods in Applied Mechanics and Engineering 1990; 79(2):173-202.

[12] Ogden RW, Roxburgh DG. A pseudo-elastic model for the Mullins effect in filled rubber. Proceedings: Mathematical, Physical and Engineering Sciences 1999; 455(1988):2861-2877. 